\title{
Newton-Kantorovich convergence theorem of a new modified Halley's method family in a Banach space
}

\author{
Rongfei Lin ${ }^{1 *}$, Yueqing Zhao ${ }^{1}$, Zdeněk Šmarda ${ }^{2}$, Qingbiao $\mathrm{Wu}^{3}$ and Yasir Khan ${ }^{3}$
}

\footnotetext{
"Correspondence: linrfei@tzc.edu.cn 'Department of Mathematics, Taizhou University, Linhai, Zhejiang 317000 , P.R. China

Full list of author information is available at the end of the article
}

\begin{abstract}
A Newton-Kantorovich convergence theorem of a new modified Halley's method family is established in a Banach space to solve nonlinear operator equations. We also present the main results to reveal the competence of our method. Finally, two numerical examples arising in the theory of the radiative transfer, neutron transport and in the kinetic theory of gasses are provided to show the application of our theorem.
\end{abstract}

\section{Introduction}

In the last two centuries, remarkable contributions have been made to both the theory and application of nonlinear equations. Suppose that we have to find a solution of the nonlinear equation

$$
F(x)=0,
$$

where $F$ is defined on an open convex subset $\Omega$ of a Banach space $X$ with values in a Banach space $Y$.

These equations are increasingly used to model problems in engineering applications, such as material science, electrical engineering, civil engineering, chemical engineering, mechanics and numerical optimization. There are several iterative methods [1-6] used to find a solution of nonlinear equations. One of those iterative methods is the famous Newton's method

$$
x_{n+1}=x_{n}-F^{\prime}\left(x_{n}\right)^{-1} F\left(x_{n}\right) \quad(n \geq 0)\left(x_{0} \in \Omega\right)
$$

often used to solve the nonlinear operator equation under the reasonable hypotheses. However, Newton's method is only the second-order convergence. Kantorovich presented the famous convergence result [7], and afterward, many Newton-Kantorovich-type convergence theorems have been attained [8-17]. Furthermore, many deformed methods [1822] have been presented to improve the convergence order. The famous Halley's method, which has been widely discussed [23-30], is the third-order convergence. The famous Halley's method is defined as

$$
x_{n+1}=x_{n}-\left[I+\frac{1}{2} L_{F}\left(x_{n}\right)\left(I-\frac{1}{2} L_{F}\left(x_{n}\right)\right)^{-1}\right] F^{\prime}\left(x_{n}\right)^{-1} F\left(x_{n}\right), \quad n=0,1, \ldots,
$$

๑ 2013 Lin et al.; licensee Springer. This is an Open Access article distributed under the terms of the Creative Commons Attribution License (http://creativecommons.org/licenses/by/2.0), which permits unrestricted use, distribution, and reproduction in any medium, provided the original work is properly cited. 
where

$$
L_{F}(x)=F^{\prime}(x)^{-1} F^{\prime \prime}(x) F^{\prime}(x)^{-1} F(x), \quad x \in \Omega .
$$

Now, we consider Halley's method with a parameter $\lambda$ in the form

$$
x_{\lambda, n+1}=x_{\lambda, n}-\left[I+\frac{1}{2} L_{F}\left(x_{\lambda, n}\right)\left(I-\lambda L_{F}\left(x_{\lambda, n}\right)\right)^{-1}\right] F^{\prime}\left(x_{\lambda, n}\right)^{-1} F\left(x_{\lambda, n}\right), \quad n=0,1, \ldots
$$

One can see that Halley's method and super-Halley's method are the special cases for $\lambda=\frac{1}{2}$ and $\lambda=1$. In this method, in every step, one needs to compute the second order derivatives of the function $F$. The computing cost will be the high. To avoid the computation of $F^{\prime \prime}\left(x_{n}\right)$, and to maintain the high order convergence, many researchers have replaced the second order derivative with the first order divided differences. They presented the modified Halley's methods with the parameters $p, \lambda$. Their modified Halley's method is as follows [12]:

$$
\left\{\begin{array}{l}
y_{n}=x_{n}-F^{\prime}\left(x_{n}\right)^{-1} F\left(x_{n}\right), \\
H\left(x_{n}, y_{n}\right)=\frac{1}{p} F^{\prime}\left(x_{n}\right)^{-1}\left[F^{\prime}\left(x_{n}+p\left(y_{n}-x_{n}\right)\right)-F^{\prime}\left(x_{n}\right)\right], \quad \lambda \in[0,1], p \in(0,1], \\
x_{n+1}=y_{n}-\frac{1}{2} H\left(x_{n}, y_{n}\right)\left[I-\lambda H\left(x_{n}, y_{n}\right)\right]\left(y_{n}-x_{n}\right) .
\end{array}\right.
$$

For $p=\frac{1}{2}, \lambda=0$, the method becomes Chebyshev's iterative method (see [13]). For $p=\frac{2}{3}$, $\lambda=1$, the method becomes inverse-free Jarratt iterative method (see $[14,15]$ ). In this paper, we establish a Kantorovich-type third-order convergence theorem for this kind of method by using majorizing function to improve the result [12].

\section{Main results}

In this section, we establish a Newton-Kantorovich convergence theorem via majorizing function. Let $g(t)=\frac{1}{6} K t^{3}+\frac{1}{2} \gamma t^{2}-t+\eta$, where $K, \gamma, \eta$ are positive real numbers. Denote

$$
\alpha=\frac{2}{\gamma+\sqrt{\gamma^{2}+2 K}}, \quad \beta=\alpha-\frac{1}{6} K \alpha^{3}-\frac{1}{2} \gamma \alpha^{2}=\frac{2\left(\gamma+2 \sqrt{\gamma^{2}+2 K}\right)}{3\left(\gamma+\sqrt{\gamma^{2}+2 K}\right)^{2}} .
$$

Theorem 1 Suppose that $X$ and $Y$ are the Banach spaces, and $\Omega$ is an open convex subset of $X, F: \Omega \subset X \rightarrow Y$ has the second order Fréchet derivative, $F^{\prime}\left(x_{0}\right)^{-1}$ exists for $x_{0} \in \Omega$, and the following conditions hold:

$$
\begin{aligned}
& \left\|F^{\prime}\left(x_{0}\right)^{-1} F\left(x_{0}\right)\right\| \leq \eta, \quad\left\|F^{\prime}\left(x_{0}\right)^{-1} F^{\prime \prime}\left(x_{0}\right)\right\| \leq \gamma, \\
& \left\|F^{\prime}\left(x_{0}\right)^{-1}\left(F^{\prime \prime}(x)-F^{\prime \prime}(y)\right)\right\| \leq N\|x-y\|, \quad x, y \in \Omega, \\
& \frac{2+3 p}{2-3 p} N \leq K, \quad \eta<\beta, \\
& \overline{S\left(x_{0}, r_{1}\right)} \subset \Omega,
\end{aligned}
$$

where $r_{1} \leq r_{2}$ are two positive real roots of the function $g(t)$. Then, for $0<p<\frac{2}{3}$, the sequence $\left\{x_{n}\right\}_{n \geq 0}$ generated by (3) is well defined, $x_{n} \in \overline{S\left(x_{0}, r_{1}\right)}$ and converges to the unique solution $x^{*}$ of equation (1) in $S\left(x_{0}, \alpha\right)$. 
Theorem 2 Suppose that $X$ and $Y$ are the Banach spaces, and $\Omega$ is an open convex subset of $X, F: \Omega \subset X \rightarrow Y$ has the third-order Fréchet derivative, $F^{\prime}\left(x_{0}\right)^{-1}$ exists for $x_{0} \in \Omega$, and the following conditions hold:

$$
\begin{aligned}
& \left\|F^{\prime}\left(x_{0}\right)^{-1} F\left(x_{0}\right)\right\| \leq \eta, \quad\left\|F^{\prime}\left(x_{0}\right)^{-1} F^{\prime \prime}\left(x_{0}\right)\right\| \leq \gamma, \quad\left\|F^{\prime}\left(x_{0}\right)^{-1} F^{\prime \prime \prime}(x)\right\| \leq N, \\
& \left\|F^{\prime}\left(x_{0}\right)^{-1}\left(F^{\prime \prime \prime}(x)-F^{\prime \prime \prime}(y)\right)\right\| \leq L\|x-y\|, \quad x, y \in \Omega, \\
& \frac{\left(1+2 p^{2}\right) L}{6 \gamma(1-p)}+N \leq K, \quad \eta<\beta, \\
& \overline{S\left(x_{0}, r_{1}\right)} \subset \Omega .
\end{aligned}
$$

Then for $0<p \leq \frac{2}{3}$, the sequence $\left\{x_{n}\right\}_{n \geq 0}$ generated by (3) is well defined, $x_{n} \in \overline{S\left(x_{0}, r_{1}\right)}$ and converges to the unique solution $x^{*}$ of equation (1) in $S\left(x_{0}, \alpha\right)$.

To prove Theorems 1 and 2, we first give some lemmas.

Lemma 1 If $\eta \leq \beta$, the polynomial $g(t)$ has two positive real roots $r_{1}, r_{2}$ (let $0<r_{1}<r_{2}<$ $+\infty)$, and a negative real root $-r_{0}\left(r_{0}>0\right)$.

Proof From definition of the function $g(t)$, there follows that $g(0)=\eta>0, \lim _{t \rightarrow-\infty} g(t)=$ $-\infty$, hence $g(t)$ has a negative root. Denote it $-r_{0}$. We get that $g^{\prime}(t)=\frac{1}{2} K t^{2}+\gamma t-1$ has the unique positive root $\alpha=\frac{2}{\gamma+\sqrt{\gamma^{2}+2 K}}$, and for $t \geq 0, g^{\prime \prime}(t)=K t+\gamma>0$. So, the necessary and sufficient condition that $g(t)$ has two positive roots for $t \geq 0$ is that the minimum of $g(t)$ satisfies $g(\alpha) \leq 0$, that is also $\eta \leq \beta$. This completes the proof of Lemma 1 .

Lemma 2 (see [12]) Suppose that the sequences $\left\{t_{n}\right\}_{n \geq 0}$ and $\left\{s_{n}\right\}_{n \geq 0}$ are generated by the following iteration $t_{0}=0$,

$$
\left\{\begin{array}{l}
s_{n}=t_{n}-g^{\prime}\left(t_{n}\right)^{-1} g\left(t_{n}\right), \\
H_{g}\left(t_{n}, s_{n}\right)=\frac{1}{p} g^{\prime}\left(t_{n}\right)^{-1}\left[g^{\prime}\left(t_{n}+p\left(s_{n}-t_{n}\right)\right)-g^{\prime}\left(t_{n}\right)\right], \\
t_{n+1}=s_{n}-\frac{1}{2} H_{g}\left(t_{n}, s_{n}\right)\left[I-\lambda H_{g}\left(t_{n}, s_{n}\right)\right]\left(s_{n}-t_{n}\right) .
\end{array}\right.
$$

Then for $\eta \leq \beta,\left\{t_{n}\right\},\left\{s_{n}\right\}$ are increasing and converge to $r_{1}$.

Lemma 3 Suppose that $F(x)$ satisfies conditions (4) of Theorem $1, \forall x \in B\left(x_{0}, r_{1}\right), F^{\prime}(x)^{-1}$ exists and satisfies the following inequalities:

(I) $\left\|F^{\prime}(x)^{-1} F^{\prime}\left(x_{0}\right)\right\| \leq-g^{\prime}\left(\left\|x-x_{0}\right\|\right)^{-1}$,

(II) $\left\|F^{\prime}\left(x_{0}\right)^{-1} F^{\prime \prime}(x)\right\| \leq g^{\prime \prime}\left(\left\|x-x_{0}\right\|\right)$.

Proof

$$
\begin{aligned}
\left\|F^{\prime}\left(x_{0}\right)^{-1} F^{\prime \prime}(x)\right\| & =\left\|F^{\prime}\left(x_{0}\right)^{-1} F^{\prime \prime}\left(x_{0}\right)+F^{\prime}\left(x_{0}\right)^{-1}\left[F^{\prime \prime}(x)-F^{\prime \prime}\left(x_{0}\right)\right]\right\| \\
& \leq \gamma+N\left\|x-x_{0}\right\| \leq \gamma+K\left\|x-x_{0}\right\|=g^{\prime \prime}\left(\left\|x-x_{0}\right\|\right) .
\end{aligned}
$$


By the proof process of Lemma 1 , we get $g^{\prime}(t)<0, t \in\left[0, r_{1}\right)$. Hence, for $x \in B\left(x_{0}, r_{1}\right)$,

$$
\begin{aligned}
& \left\|F^{\prime}\left(x_{0}\right)^{-1} F^{\prime}(x)-I\right\| \\
& \quad=\left\|F^{\prime}\left(x_{0}\right)^{-1}\left[F^{\prime}(x)-F^{\prime}\left(x_{0}\right)-F^{\prime \prime}\left(x_{0}\right)\left(x-x_{0}\right)+F^{\prime \prime}\left(x_{0}\right)\left(x-x_{0}\right)\right]\right\| \\
& \quad \leq\left\|\int_{0}^{1} F^{\prime}\left(x_{0}\right)^{-1}\left[F^{\prime \prime}\left(x_{0}+t\left(x-x_{0}\right)\right)-F^{\prime \prime}\left(x_{0}\right)\right] d t\left(x-x_{0}\right)\right\|+\gamma\left\|x-x_{0}\right\| \\
& \quad \leq \int_{0}^{1} N t d t\left\|x-x_{0}\right\|^{2}+\gamma\left\|x-x_{0}\right\| \leq \frac{1}{2} K\left\|x-x_{0}\right\|^{2}+\gamma\left\|x-x_{0}\right\| \\
& \quad=1+g^{\prime}\left(\left\|x-x_{0}\right\|\right)<1 .
\end{aligned}
$$

By the Banach theorem, we know $\left(F^{\prime}\left(x_{0}\right)^{-1} F^{\prime}(x)\right)^{-1}=F^{\prime}(x)^{-1} F^{\prime}\left(x_{0}\right)$ exists, and

$$
\left\|F^{\prime}(x)^{-1} F^{\prime}\left(x_{0}\right)\right\| \leq \frac{1}{1-\left\|I-F^{\prime}\left(x_{0}\right)^{-1} F^{\prime}(x)\right\|} \leq-g^{\prime}\left(\left\|x-x_{0}\right\|\right)^{-1} .
$$

This completes the proof of Lemma 3.

Lemma 4 Suppose that the nonlinear operator $F: \Omega \subset X \rightarrow Y$ is defined on an open convex subset $\Omega$ of a Banach space $X$ with values in a Banach space $Y$, F has the second-order Frechét derivative, and the sequences $\left\{x_{n}\right\},\left\{y_{n}\right\}$ are generated by (3). Then the following formula holds for all natural numbers $n$ :

$$
\begin{aligned}
F\left(x_{n+1}\right)= & \int_{0}^{1} F^{\prime \prime}\left(y_{n}+t\left(x_{n+1}-y_{n}\right)\right)(1-t) d t\left(x_{n+1}-y_{n}\right)^{2} \\
& +\int_{0}^{1}\left[F^{\prime \prime}\left(x_{n}+t\left(y_{n}-x_{n}\right)\right)(1-t)-\frac{1}{2} F^{\prime \prime}\left(x_{n}+p t\left(y_{n}-x_{n}\right)\right)\right] d t\left(y_{n}-x_{n}\right)^{2} \\
& -\frac{1-\lambda}{2} \int_{0}^{1} F^{\prime \prime}\left(x_{n}+p t\left(y_{n}-x_{n}\right)\right) d t\left(y_{n}-x_{n}\right) H\left(x_{n}, y_{n}\right)\left(y_{n}-x_{n}\right) \\
& -\frac{1}{2} \int_{0}^{1}\left[F^{\prime \prime}\left(x_{n}+t\left(y_{n}-x_{n}\right)\right)-F^{\prime \prime}\left(x_{n}+p t\left(y_{n}-x_{n}\right)\right)\right] d t\left(y_{n}-x_{n}\right) \\
& \times H\left(x_{n}, y_{n}\right)\left(y_{n}-x_{n}\right) \\
& +\frac{\lambda}{2} \int_{0}^{1} F^{\prime \prime}\left(x_{n}+t\left(y_{n}-x_{n}\right)\right) d t\left(y_{n}-x_{n}\right) H\left(x_{n}, y_{n}\right) H\left(x_{n}, y_{n}\right)\left(y_{n}-x_{n}\right) .
\end{aligned}
$$

Proof

$$
\begin{aligned}
& F\left(x_{n+1}\right)=F\left(x_{n+1}\right)-F\left(y_{n}\right)-F^{\prime}\left(y_{n}\right)\left(x_{n+1}-y_{n}\right)+F\left(y_{n}\right)+F^{\prime}\left(y_{n}\right)\left(x_{n+1}-y_{n}\right) \\
& =\int_{0}^{1} F^{\prime \prime}\left(y_{n}+t\left(x_{n+1}-y_{n}\right)\right)(1-t) d t\left(x_{n+1}-y_{n}\right)^{2}+F\left(y_{n}\right)+F\left(y_{n}^{\prime}\right)\left(x_{n+1}-y_{n}\right), \\
& F^{\prime}\left(x_{n}\right) H\left(x_{n}, y_{n}\right)=\frac{1}{p}\left[F^{\prime}\left(x_{n}+p\left(y_{n}-x_{n}\right)\right)-F^{\prime}\left(x_{n}\right)\right]=\int_{0}^{1} F^{\prime \prime}\left(x_{n}+p t\left(y_{n}-x_{n}\right)\right) d t\left(y_{n}-x_{n}\right), \\
& F\left(y_{n}\right)+F\left(y_{n}^{\prime}\right)\left(x_{n+1}-y_{n}\right) \\
& =F\left(y_{n}\right)-F\left(x_{n}\right)-F^{\prime}\left(x_{n}\right)\left(y_{n}-x_{n}\right)^{2}-\frac{1}{2} F^{\prime}\left(y_{n}\right) H\left(x_{n}, y_{n}\right)\left[I-\lambda H\left(x_{n}, y_{n}\right)\right]\left(y_{n}-x_{n}\right)
\end{aligned}
$$




$$
\begin{aligned}
= & \int_{0}^{1} F^{\prime \prime}\left(x_{n}+t\left(y_{n}-x_{n}\right)\right)(1-t) d t\left(y_{n}-x_{n}\right)^{2} \\
& -\frac{1}{2}\left[F^{\prime}\left(y_{n}\right)-F^{\prime}\left(x_{n}\right)\right] H\left(x_{n}, y_{n}\right)\left[I-\lambda H\left(x_{n}, y_{n}\right)\right]\left(y_{n}-x_{n}\right) \\
& -\frac{1}{2} F^{\prime}\left(x_{n}\right) H\left(x_{n}, y_{n}\right)\left[I-\lambda H\left(x_{n}, y_{n}\right)\right]\left(y_{n}-x_{n}\right) \\
= & \int_{0}^{1} F^{\prime \prime}\left(x_{n}+t\left(y_{n}-x_{n}\right)\right)(1-t) d t\left(y_{n}-x_{n}\right)^{2}-\frac{1}{2} \int_{0}^{1} F^{\prime \prime}\left(x_{n}+p t\left(y_{n}-x_{n}\right)\right) d t\left(y_{n}-x_{n}\right)^{2} \\
& +\frac{\lambda}{2} \int_{0}^{1} F^{\prime \prime}\left(x_{n}+p t\left(y_{n}-x_{n}\right)\right) d t\left(y_{n}-x_{n}\right) H\left(x_{n}, y_{n}\right)\left(y_{n}-x_{n}\right) \\
& -\frac{1}{2} \int_{0}^{1} F^{\prime \prime}\left(x_{n}+t\left(y_{n}-x_{n}\right)\right) d t\left(y_{n}-x_{n}\right) H\left(x_{n}, y_{n}\right)\left(y_{n}-x_{n}\right) \\
& +\frac{\lambda}{2} \int_{0}^{1} F^{\prime \prime}\left(x_{n}+t\left(y_{n}-x_{n}\right)\right) d t\left(y_{n}-x_{n}\right) H\left(x_{n}, y_{n}\right) H\left(x_{n}, y_{n}\right)\left(y_{n}-x_{n}\right) .
\end{aligned}
$$

Hence,

$$
\begin{aligned}
F\left(x_{n+1}\right)= & \int_{0}^{1} F^{\prime \prime}\left(y_{n}+t\left(x_{n+1}-y_{n}\right)\right)(1-t) d t\left(x_{n+1}-y_{n}\right)^{2} \\
& +\int_{0}^{1}\left[F^{\prime \prime}\left(x_{n}+t\left(y_{n}-x_{n}\right)\right)(1-t)-\frac{1}{2} F^{\prime \prime}\left(x_{n}+p t\left(y_{n}-x_{n}\right)\right)\right] d t\left(y_{n}-x_{n}\right)^{2} \\
& -\frac{1-\lambda}{2} \int_{0}^{1} F^{\prime \prime}\left(x_{n}+p t\left(y_{n}-x_{n}\right)\right) d t\left(y_{n}-x_{n}\right) H\left(x_{n}, y_{n}\right)\left(y_{n}-x_{n}\right) \\
& -\frac{1}{2} \int_{0}^{1}\left[F^{\prime \prime}\left(x_{n}+t\left(y_{n}-x_{n}\right)\right)-F^{\prime \prime}\left(x_{n}+p t\left(y_{n}-x_{n}\right)\right)\right] d t\left(y_{n}-x_{n}\right) \\
& \times H\left(x_{n}, y_{n}\right)\left(y_{n}-x_{n}\right) \\
& +\frac{\lambda}{2} \int_{0}^{1} F^{\prime \prime}\left(x_{n}+t\left(y_{n}-x_{n}\right)\right) d t\left(y_{n}-x_{n}\right) H\left(x_{n}, y_{n}\right) H\left(x_{n}, y_{n}\right)\left(y_{n}-x_{n}\right) .
\end{aligned}
$$

This completes the proof of Lemma 4.

Proof of Theorem 1 By induction, we can prove, for $n \geq 0$, that the following formulae hold:

$$
\begin{array}{ll}
\left(\mathrm{I}_{n}\right): & x_{n} \in \overline{S\left(x_{0}, t_{n}\right)} \\
\left(\mathrm{II}_{n}\right): & \left\|F^{\prime}\left(x_{n}\right)^{-1} F^{\prime}\left(x_{0}\right)\right\| \leq-g^{\prime}\left(t_{n}\right)^{-1}, \quad\left\|F^{\prime}\left(x_{0}\right)^{-1} F^{\prime \prime}\left(x_{n}\right)\right\| \leq g^{\prime \prime}\left(t_{n}\right) \\
\left(\mathrm{III}_{n}\right): & \left\|y_{n}-x_{n}\right\| \leq s_{n}-t_{n} \\
\left(\mathrm{IV}_{n}\right): & y_{n} \in \overline{S\left(x_{0}, s_{n}\right)} \\
\left(\mathrm{V}_{n}\right): & \left\|x_{n+1}-y_{n}\right\| \leq t_{n+1}-s_{n} .
\end{array}
$$

In fact, by Lemma 2, we know that $\left\{t_{n}\right\}$ is increasing and converges to the minimum positive root of the function $g(t)$. Hence, $t_{n}<r_{1}$ for all natural numbers $n$. It is easy to verify it for the case $n=0$. By using mathematical induction, we now suppose the formulae above also hold for $n \geq 0$. Then

$$
\left(\mathrm{I}_{n+1}\right): \quad\left\|x_{n+1}-x_{0}\right\| \leq\left\|x_{n+1}-y_{n}\right\|+\left\|y_{n}-x_{0}\right\| \leq t_{n+1}-s_{n}+s_{n}=t_{n+1} .
$$


By Lemma 3, and the fact that $-g^{\prime}(t)^{-1}, g^{\prime \prime}(t)$ are increasing on $\left[0, r_{1}\right]$, we get $\left(\mathrm{II}_{n+1}\right)$.

$$
\begin{aligned}
& \left(\mathrm{III}_{n+1}\right): \| \int_{0}^{1} F^{\prime}\left(x_{0}\right)^{-1}\left[F^{\prime \prime}\left(y_{n}+t\left(x_{n+1}-y_{n}\right)\right)(1-t) d t\left(x_{n+1}-y_{n}\right)^{2} \|\right. \\
& \leq \int_{0}^{1} g^{\prime \prime}\left(\left\|y_{n}-x_{0}+t\left(x_{n+1}-y_{n}\right)\right\|\right)(1-t) d t\left(t_{n+1}-s_{n}\right)^{2} \\
& \leq \int_{0}^{1} g^{\prime \prime}\left(s_{n}+t\left(t_{n+1}-s_{n}\right)\right)(1-t) d t\left(t_{n+1}-s_{n}\right)^{2} \\
& \left\|\int_{0}^{1} F^{\prime}\left(x_{0}\right)^{-1}\left[F^{\prime \prime}\left(x_{n}+t\left(y_{n}-x_{n}\right)\right)(1-t)-\frac{1}{2} F^{\prime \prime}\left(x_{n}+p t\left(y_{n}-x_{n}\right)\right)\right] d t\right\| \\
& \leq\left\|\int_{0}^{1} F^{\prime}\left(x_{0}\right)^{-1}\left[F^{\prime \prime}\left(x_{n}+t\left(y_{n}-x_{n}\right)\right)-F^{\prime \prime}\left(x_{n}\right)\right](1-t) d t\right\| \\
& +\frac{1}{2}\left\|\int_{0}^{1} F^{\prime}\left(x_{0}\right)^{-1}\left[F^{\prime \prime}\left(x_{n}+p t\left(y_{n}-x_{n}\right)\right)-F^{\prime \prime}\left(x_{n}\right)\right] d t\right\| \\
& \leq \frac{(2+3 p) N}{12}\left\|y_{n}-x_{n}\right\| \\
& \int_{0}^{1} F^{\prime}\left(x_{0}\right)^{-1}\left[F^{\prime \prime}\left(x_{n}+t\left(y_{n}-x_{n}\right)\right)-F^{\prime \prime}\left(x_{n}+p t\left(y_{n}-x_{n}\right)\right)\right] d t \\
& \leq \int_{0}^{1} N(1-p) t\left\|y_{n}-x_{n}\right\| d t \leq \frac{(1-p) N}{2}\left(s_{n}-t_{n}\right) \\
& \left\|H\left(x_{n}, y_{n}\right)\right\|=\left\|F^{\prime}\left(x_{n}\right)^{-1} \int_{0}^{1} F^{\prime \prime}\left(x_{n}+p t\left(y_{n}-x_{n}\right)\right)\left(y_{n}-x_{n}\right) d t\right\| \\
& =\left\|F^{\prime}\left(x_{n}\right)^{-1} F^{\prime}\left(x_{0}\right) \int_{0}^{1} F^{\prime}\left(x_{0}\right)^{-1} F^{\prime \prime}\left(x_{n}+p t\left(y_{n}-x_{n}\right)\right)\left(y_{n}-x_{n}\right) d t\right\| \\
& \leq-g^{\prime}\left(t_{n}\right)^{-1} \int_{0}^{1} g^{\prime \prime}\left(t_{n}+p t\left(s_{n}-t_{n}\right)\right) d t\left(s_{n}-t_{n}\right)=-H_{g}\left(t_{n}, s_{n}\right) \text {. }
\end{aligned}
$$

From Lemmas 3 and 4, we get

$$
\begin{aligned}
& \left\|F^{\prime}\left(x_{0}\right)^{-1} F\left(x_{n+1}\right)\right\| \leq \int_{0}^{1} g^{\prime \prime}\left(s_{n}+t\left(t_{n+1}-s_{n}\right)\right)(1-t) d t\left(t_{n+1}-s_{n}\right)^{2}+\frac{(2+3 p) N}{12}\left(s_{n}-t_{n}\right)^{3} \\
& +\frac{1-\lambda}{2} \int_{0}^{1} g^{\prime \prime}\left(t_{n}+p t\left(s_{n}-t_{n}\right)\right) d t\left(-H_{g}\left(t_{n}, s_{n}\right)\right)\left(s_{n}-t_{n}\right)^{2} \\
& +\frac{1}{2} \frac{(1-p) N}{2}\left(-H_{g}\left(t_{n}, s_{n}\right)\right)\left(s_{n}-t_{n}\right)^{3} \\
& +\frac{\lambda}{2} \int_{0}^{1} g^{\prime \prime}\left(t_{n}+t\left(s_{n}-t_{n}\right)\right) d t\left(s_{n}-t_{n}\right)^{2} H_{g}^{2}\left(t_{n}, s_{n}\right) \\
& \leq \int_{0}^{1} g^{\prime \prime}\left(s_{n}+t\left(t_{n+1}-s_{n}\right)\right)(1-t) d t\left(t_{n+1}-s_{n}\right)^{2} \\
& +\frac{(2-3 p)}{12} \cdot \frac{2+3 p}{2-3 p} N\left(s_{n}-t_{n}\right)^{3} \\
& -\frac{1-\lambda}{2} \int_{0}^{1} g^{\prime \prime}\left(t_{n}+p t\left(s_{n}-t_{n}\right)\right) d t H_{g}\left(t_{n}, s_{n}\right)\left(s_{n}-t_{n}\right)^{2} \\
& -\frac{1}{2} \frac{(1-p) K}{2} H_{g}\left(t_{n}, s_{n}\right)\left(s_{n}-t_{n}\right)^{3} \\
& +\frac{\lambda}{2} \int_{0}^{1} g^{\prime \prime}\left(t_{n}+t\left(s_{n}-t_{n}\right)\right) d t\left(s_{n}-t_{n}\right)^{2} H_{g}^{2}\left(t_{n}, s_{n}\right)
\end{aligned}
$$




$$
\begin{aligned}
\leq & \int_{0}^{1} g^{\prime \prime}\left(s_{n}+t\left(t_{n+1}-s_{n}\right)\right)(1-t) d t\left(t_{n+1}-s_{n}\right)^{2}+\frac{(2-3 p) K}{12}\left(s_{n}-t_{n}\right)^{3} \\
& -\frac{1-\lambda}{2} \int_{0}^{1} g^{\prime \prime}\left(t_{n}+p t\left(s_{n}-t_{n}\right)\right) d t H_{g}\left(t_{n}, s_{n}\right)\left(s_{n}-t_{n}\right)^{2} \\
& -\frac{1}{2} \frac{(1-p) K}{2} H_{g}\left(t_{n}, s_{n}\right)\left(s_{n}-t_{n}\right)^{3} \\
& +\frac{\lambda}{2} \int_{0}^{1} g^{\prime \prime}\left(t_{n}+t\left(s_{n}-t_{n}\right)\right) d t\left(s_{n}-t_{n}\right)^{2} H_{g}^{2}\left(t_{n}, s_{n}\right) \\
= & g\left(t_{n+1}\right) .
\end{aligned}
$$

Hence, we get

$$
\begin{aligned}
&\left\|y_{n+1}-x_{n+1}\right\| \leq\left\|-F^{\prime}\left(x_{n+1}\right)^{-1} F^{\prime}\left(x_{0}\right)\right\|\left\|F^{\prime}\left(x_{0}\right)^{-1} F\left(x_{n+1}\right)\right\| \\
& \leq-g^{\prime}\left(t_{n+1}\right)^{-1} g\left(t_{n+1}\right)=s_{n+1}-t_{n+1}, \\
&\left(\mathrm{IV}_{n+1}\right):\left\|y_{n+1}-x_{0}\right\| \leq\left\|y_{n+1}-x_{n+1}\right\|+\left\|x_{n+1}-x_{0}\right\| \leq\left(s_{n+1}-t_{n+1}\right)+t_{n+1}=s_{n+1}, \\
&\left(\mathrm{~V}_{n+1}\right):\left\|x_{n+2}-y_{n+1}\right\|=\left\|-\frac{1}{2} H\left(x_{n+1}, y_{n+1}\right)\left[I-\lambda H\left(x_{n+1}, y_{n+1}\right)\right]\left(y_{n+1}-x_{n+1}\right)\right\| \\
& \leq \frac{1}{2}\left\|H\left(x_{n+1}, y_{n+1}\right)\right\|\left[1+\lambda\left\|H\left(x_{n+1}, y_{n+1}\right)\right\|\right]\left\|\left(y_{n+1}-x_{n+1}\right)\right\| \\
& \leq-\frac{1}{2} H_{g}\left(t_{n+1}, s_{n+1}\right)\left[1-\lambda H_{g}\left(t_{n+1}, s_{n+1}\right)\right]\left(s_{n+1}-t_{n+1}\right) \\
&=t_{n+2}-s_{n+1} .
\end{aligned}
$$

So, the sequence $\left\{x_{n}\right\}_{n \geq 0}$ generated by (3) is well defined, $x_{n} \in \overline{S\left(x_{0}, r_{1}\right)}$ and $\left\{x_{n}\right\}$ converges to the solution $x^{*}$ of equation (1) on $\overline{S\left(x_{0}, r_{1}\right)}$. Now, we prove the uniqueness. If $y^{*}$ is also the solution of equation (1) in $S\left(x_{0}, \alpha\right)$, then, by the proof of Lemma 1, we know that $g^{\prime}(t)<0$, $t \in[0, \alpha)$.

Thus,

$$
\begin{aligned}
& \left\|F^{\prime}\left(x_{0}\right)^{-1} \int_{0}^{1} F^{\prime}\left(x^{*}+t\left(y^{*}-x^{*}\right)\right) d t-I\right\| \\
& \quad=\left\|\int_{0}^{1} F^{\prime}\left(x_{0}\right)^{-1}\left\{F^{\prime}\left[x^{*}+t\left(y^{*}-x^{*}\right)\right]-F^{\prime}\left(x_{0}\right)\right\} d t\right\| \\
& \leq \int_{0}^{1} \int_{0}^{1}\left\|F^{\prime}\left(x_{0}\right)^{-1} F^{\prime \prime}\left\{x_{0}+s\left[x^{*}+t\left(y^{*}-x^{*}\right)-x_{0}\right]\right\}\right\|\left\|x^{*}-x_{0}+t\left(y^{*}-x^{*}\right)\right\| d s d t \\
& \leq \int_{0}^{1} \int_{0}^{1} g^{\prime \prime}\left[s\left\|x^{*}-x_{0}+t\left(y^{*}-x^{*}\right)\right\|\right]\left\|x^{*}-x_{0}+t\left(y^{*}-x^{*}\right)\right\| d s d t \\
& =\int_{0}^{1}\left\{g^{\prime}\left[\left\|x^{*}-x_{0}+t\left(y^{*}-x^{*}\right)\right\|\right]-g^{\prime}(0)\right\} d t \\
& =\int_{0}^{1}\left\{g^{\prime}\left[\left\|(1-t)\left(x^{*}-x_{0}\right)+t\left(y^{*}-x_{0}\right)\right\|\right]\right\} d t+1<1 .
\end{aligned}
$$

By the Banach theorem, we know the inverse of

$$
\int_{0}^{1} F^{\prime}\left[x^{*}+t\left(y^{*}-x^{*}\right)\right] d t
$$


exists. Since

$$
0=F\left(y^{*}\right)-F\left(x^{*}\right)=\int_{0}^{1} F^{\prime}\left[x^{*}+t\left(y^{*}-x^{*}\right)\right] d t\left(y^{*}-x^{*}\right)
$$

we have $y^{*}=x^{*}$. This completes the proof of uniqueness. Thus, the proof of Theorem 1 is complete.

Proof of Theorem 2 We know that $F: \Omega \subset X \rightarrow Y$ has the three-order Fréchet derivative.

Then

$$
\begin{aligned}
& -H_{g}\left(x_{n}, y_{n}\right)=-g^{\prime}\left(t_{n}\right)^{-1} \int_{0}^{1} g^{\prime \prime}\left(t_{n}+p t\left(s_{n}-t_{n}\right)\right) d t\left(s_{n}-t_{n}\right) \\
& =\frac{1}{1-\gamma t_{n}-\frac{1}{2} t_{n}^{2}} \int_{0}^{1}\left[K\left(t_{n}+p t\left(s_{n}-t_{n}\right)\right)+\gamma\right] d t\left(s_{n}-t_{n}\right) \geq \gamma\left(s_{n}-t_{n}\right)>0, \\
& \left\|\int_{0}^{1} F^{\prime}\left(x_{0}\right)^{-1}\left[F^{\prime \prime}\left(x_{n}+t\left(y_{n}-x_{n}\right)\right)(1-t)-\frac{1}{2} F^{\prime \prime}\left(x_{n}+p t\left(y_{n}-x_{n}\right)\right)\right] d t\right\| \\
& \leq\left\|\int_{0}^{1} \int_{0}^{1} F^{\prime}\left(x_{0}\right)^{-1}\left[F^{\prime \prime \prime}\left(x_{n}+\sigma t\left(y_{n}-x_{n}\right)\right)-F^{\prime \prime \prime}\left(x_{n}\right)\right] t(1-t) d \sigma d t\right\|\left\|y_{n}-x_{n}\right\| \\
& +\frac{p}{2}\left\|\int_{0}^{1} \int_{0}^{1} F^{\prime}\left(x_{0}\right)^{-1}\left[F^{\prime \prime \prime}\left(x_{n}+p \sigma t\left(y_{n}-x_{n}\right)\right)-F^{\prime \prime \prime}\left(x_{n}\right)\right] t d \sigma d t\right\|\left\|y_{n}-x_{n}\right\| \\
& +\frac{2-3 p}{12} F^{\prime}\left(x_{0}\right)^{-1} F^{\prime \prime \prime}\left(x_{n}\right)\left\|y_{n}-x_{n}\right\| \\
& \leq \frac{\left(1+2 p^{2}\right) L}{24}\left\|y_{n}-x_{n}\right\|^{2}+\frac{2-3 p}{12} N\left\|y_{n}-x_{n}\right\|, \\
& \frac{\left(1+2 p^{2}\right) L}{24}\left\|y_{n}-x_{n}\right\|^{4}+\frac{1}{2} \frac{(1-p) N}{2}\left(-H_{g}\left(t_{n}, s_{n}\right)\right)\left(s_{n}-t_{n}\right)^{3} \\
& \leq\left[\frac{\left(1+2 p^{2}\right) L}{6(1-p)} \frac{\left(s_{n}-t_{n}\right)}{\left(-H_{g}\left(t_{n}, s_{n}\right)\right)}+N\right] \frac{(1-p)}{4}\left(-H_{g}\left(t_{n}, s_{n}\right)\right)\left(s_{n}-t_{n}\right)^{3} \\
& \leq\left[\frac{\left(1+2 p^{2}\right) L}{6 \gamma(1-p)}+N\right] \frac{(1-p)}{4}\left(-H_{g}\left(t_{n}, s_{n}\right)\right)\left(s_{n}-t_{n}\right)^{3} \\
& \leq-\frac{(1-p)}{4} K H_{g}\left(t_{n}, s_{n}\right)\left(s_{n}-t_{n}\right)^{3} \text {. }
\end{aligned}
$$

Hence,

$$
\begin{aligned}
\left\|F^{\prime}\left(x_{0}\right)^{-1} F\left(x_{n+1}\right)\right\| \leq & \int_{0}^{1} g^{\prime \prime}\left(s_{n}+t\left(t_{n+1}-s_{n}\right)\right)(1-t) d t\left(t_{n+1}-s_{n}\right)^{2} \\
& +\frac{\left(1+2 p^{2}\right) L}{24}\left\|y_{n}-x_{n}\right\|^{4}+\frac{2-3 p}{12} N\left\|y_{n}-x_{n}\right\|^{3} \\
& +\frac{1-\lambda}{2} \int_{0}^{1} g^{\prime \prime}\left(t_{n}+p t\left(s_{n}-t_{n}\right)\right) d t\left(-H_{g}\left(t_{n}, s_{n}\right)\right)\left(s_{n}-t_{n}\right)^{2} \\
& +\frac{1}{2} \frac{(1-p) N}{2}\left(-H_{g}\left(t_{n}, s_{n}\right)\right)\left(s_{n}-t_{n}\right)^{3} \\
& +\frac{\lambda}{2} \int_{0}^{1} g^{\prime \prime}\left(t_{n}+t\left(s_{n}-t_{n}\right)\right) d t\left(s_{n}-t_{n}\right)^{2} H_{g}^{2}\left(t_{n}, s_{n}\right)
\end{aligned}
$$




$$
\begin{aligned}
\leq & \int_{0}^{1} g^{\prime \prime}\left(s_{n}+t\left(t_{n+1}-s_{n}\right)\right)(1-t) d t\left(t_{n+1}-s_{n}\right)^{2}+\frac{(2-3 p)}{12} K\left(s_{n}-t_{n}\right)^{3} \\
& -\frac{1-\lambda}{2} \int_{0}^{1} g^{\prime \prime}\left(t_{n}+p t\left(s_{n}-t_{n}\right)\right) d t H_{g}\left(t_{n}, s_{n}\right)\left(s_{n}-t_{n}\right)^{2} \\
& -\frac{(1-p) K}{4} H_{g}\left(t_{n}, s_{n}\right)\left(s_{n}-t_{n}\right)^{3} \\
& +\frac{\lambda}{2} \int_{0}^{1} g^{\prime \prime}\left(t_{n}+t\left(s_{n}-t_{n}\right)\right) d t\left(s_{n}-t_{n}\right)^{2} H_{g}^{2}\left(t_{n}, s_{n}\right)=g\left(t_{n+1}\right) .
\end{aligned}
$$

Using the same proof method as in Theorem 1, we get assertion of Theorem 2.

\section{Numerical examples}

In this section, we apply the convergence ball result and show two numerical examples.

Example 1 Suppose that $F(x)=\frac{1}{6} x^{3}+\frac{1}{6} x^{2}-\frac{5}{6} x+\frac{1}{3}=0$, we consider initial point $x_{0}=0$, $\Omega=[-1,1]$. We can choose

$$
\eta=\gamma=\frac{2}{5}, \quad N=\frac{6}{5}, \quad L=0
$$

Hence,

$$
K=N=\frac{6}{5}, \quad \beta=\frac{2\left(\gamma+2 \sqrt{\gamma^{2}+2 K}\right)}{3\left(\gamma+\sqrt{\gamma^{2}+2 K}\right)^{2}}=\frac{3}{5}, \quad \eta<\beta .
$$

Moreover, by Theorem 2, we get that the sequence $x_{n}(n \geq 0)$ generated by (3) is well defined and convergent.

Example 2 Consider the following integral equations

$$
x(s)=1+\frac{1}{4} x(s) \int_{0}^{1} \frac{s}{s+t} x(t) d t
$$

and the space $X=C[0,1]$ with the norm

$$
\|x\|=\max _{0 \leq s \leq 1}|x(s)| .
$$

This equation arises in the theory of the radiative transfer, neutron transport and in the kinetic theory of gasses. Let us define the operator $F$ on $X$ by

$$
F(x)=\frac{1}{4} x(s) \int_{0}^{1} \frac{s}{s+t} x(t) d t-x(s)+1 .
$$

Then, for $x_{0}=1$, we get the following results:

$$
\begin{aligned}
& N=0, \quad L=0, \quad K=0, \quad\left\|F^{\prime}\left(x_{0}\right)^{-1}\right\|=1.5304, \\
& \eta=\left\|F^{\prime}\left(x_{0}\right)^{-1} F^{\prime}\left(x_{0}\right)\right\|=0.2652,
\end{aligned}
$$




$$
\begin{aligned}
& \gamma=\left\|F^{\prime}\left(x_{0}\right)^{-1} F^{\prime \prime}\left(x_{0}\right)\right\|=1.5304 \times 2 \cdot \frac{1}{4} \max _{0 \leq s \leq 1}\left|\int_{0}^{1} \frac{s}{s+t} d t\right|=0.5303, \\
& \frac{2\left(\gamma+2 \sqrt{\gamma^{2}+2 K}\right)}{3\left(\gamma+\sqrt{\gamma^{2}+2 K}\right)^{2}}=0.9429>\eta
\end{aligned}
$$

\section{this means that the hypotheses of Theorem 2 hold.}

\section{Competing interests}

The authors declare that they have no competing interests.

Authors' contributions

The authors have made the same contribution. All authors read and approved the final manuscript.

\section{Author details}

${ }^{1}$ Department of Mathematics, Taizhou University, Linhai, Zhejiang 317000, P.R. China. ${ }^{2}$ Department of Mathematics, Brno University of Technology, Brno, Czech Republic. ${ }^{3}$ Department of Mathematics, Zhejiang University, Hangzhou, Zhejiang 310027, P.R. China.

\section{Acknowledgements}

This work is supported by the National Basic Research 973 Program of China (No. 2011 JB105001), the National Natural Science Foundation of China (Grant No. 11371320), the Foundation of Science and Technology Department (Grant No. 2013C31084) of Zhejiang Province and the Foundation of the Education Department (No. 20120040, Y201329420) of Zhejiang Province of China and by the Grant FEKT-S-11-2-921 of Faculty of Electrical Engineering and Communication, Brno University of Technology, Czech Republic

\section{Received: 19 July 2013 Accepted: 4 September 2013 Published: 19 Nov 2013}

\section{References}

1. Ortega, JM, Rheinbolt, WC: Iterative Solution of Nonlinear Equations in Several Variables. Academic Press, New York (1970)

2. Cordero, A, Hueso, JL, Martinez, E, Torregrosa, JR: Increasing the convergence order of an iterative method for nonlinear systems. Appl. Math. Lett. 25, 2369-2374 (2012)

3. Babajee, DKR, Cordero, A, Soleymani, F, Torregrosa, JR: On a novel fourth-order algorithm for solving systems of nonlinear equations. J. Appl. Math. 2012, AID 165452 (2012)

4. Cordero, A, Torregrosa, JR, Vindel, P: Study of the dynamics of third-order iterative methods on quadratic polynomials. Int. J. Comput. Math. 89, 1826-1836 (2012)

5. Soleymani, F: Some efficient seventh-order derivative-free families in root-finding. Opusc. Math. 33, 163-173 (2013)

6. Soleymani, F, Karimi Vanani, S: A modified eighth-order derivative-free root solver. Thai J. Math. 10, 541-549 (2012)

7. Kantorovich, L: On Newton method. Tr. Mat. Inst. Steklova 28, 104-144 (1949)

8. Wu, QB, Zhao, YQ: Third-order convergence theorem by using majorizing function for a modified Newton method in Banach space. Appl. Math. Comput. 175, 1515-1524 (2006)

9. Ezquerro, JA, Hemández, MA: On the R-order of Halley method. J. Math. Anal. Appl. 303, 591-601 (2005)

10. Gutierrez, JM, Hernandez, MA: Recurrence relations for the Super-Halley method. Comput. Math. Appl. 36, 1-8 (1998)

11. Wu, QB, Zhao, YQ: Newton-Kantorovich-type convergence theorem for a family of new deformed Chebyshev method. Appl. Math. Comput. 192, 405-412 (2007)

12. Guo, X: The convergence for the second-order-derivative-free iterations. J. Eng. Math. 18, 29-34 (2001) (in Chinese)

13. Argyros, IK, Chen, D: Results on the Chebyshev method in Banach spaces. Proyecciones 12, 119-128 (1993)

14. Ezquerro, JA, et al.: The application of an inverse-free Jarratt-type approximation to nonlinear integral equations of Hammersteintype. Comput. Math. Appl. 36, 9-20 (1998)

15. Argyros, IK: A new convergence theorem for the Jarratt method in Banach space. Comput. Math. Appl. 36, 13-18 (1998)

16. Ezquerro, JA, Gonzalez, D, Hernandez, MA: A modification of the classic conditions of Newton-Kantorovich for Newton's method. Math. Comput. Model. 57, 584-594 (2013)

17. Ezquerro, JA, Gonzalez, D, Hernandez, MA: A variant of the Newton-Kantorovich theorem for nonlinear integral equations of mixed Hammerstein type. Appl. Math. Comput. 218, 9536-9546 (2012)

18. Homeier, $\mathrm{HHH}$ : A modified Newton method with cubic convergence: the multivariate case. J. Comput. Appl. Math. $169,161-169(2004)$

19. Weerakoon, S, Fernando, TGI: A variant of Newton's method with accelerated third-order convergence. Appl. Math. Lett. 13, 87-93 (2000)

20. Frontini, M, Sormani, E: Some variant of Newton's method with third-order convergence. Appl. Math. Comput. 140, 419-426 (2003)

21. Kou, JS, Li, YT, Wang, XH: On modified Newton methods with cubic convergence. Appl. Math. Comput. 176, 123-127 (2006)

22. Chen, M, Khan, Y, Wu, Q, Yildirim, A: Newton-Kantorovich convergence theorem of a modified Newton's method under the gamma-condition in a Banach space. J. Optim. Theory Appl. (2013). doi:10.1007/s10957-012-0237-9

23. Chen, D, Argyros, IK, Qian, QS: A note on the Halley method in Banach spaces. Appl. Math. Comput. 58, 215-224 (2004) 
24. Argyros, IK: The super-Halley method using divided differences. Appl. Math. Lett. 10, 91-95 (1997)

25. Gutiérrez, JM, Hernández, MA: Recurrence relations for the super-Halley method. Comput. Math. Appl. 36, 1-8 (1998)

26. Hernández, MA, Salanova, MA: Indices of convexity and concavity: application to Halley method. Appl. Math. Comput. 103, 27-49 (1999)

27. Ezquerro, JA, Hernández, MA: A modification of the super-Halley method under mild differentiability conditions. J. Comput. Appl. Math. 114, 405-409 (2000)

28. Ezquerro, JA, Hernández, MA: On the R-order of the Halley method. J. Comput. Appl. Math. 303, $591-601$ (2005)

29. Kou, JS, Li, YT, Wang, XH: Modified Halley's method free from second derivative. Appl. Math. Comput. 183, 704-708 (2006)

30. Gutiérrez, JM, Hernández, MA: An acceleration of Newton's method: super-Halley method. J. Appl. Math. Comput. $117,223-239(2001)$

10.1186/1687-1847-2013-325

Cite this article as: Lin et al.: Newton-Kantorovich convergence theorem of a new modified Halley's method family in a Banach space. Advances in Difference Equations 2013, 2013:325

Submit your manuscript to a SpringerOpen ${ }^{\odot}$ journal and benefit from:

- Convenient online submission

- Rigorous peer review

- Immediate publication on acceptance

- Open access: articles freely available online

- High visibility within the field

- Retaining the copyright to your article 